\title{
Constraint based modelling as a mean to link dialectical thinking and corporate data. Application to the Design of Experiments.
}

\author{
Thomas Eltzer, Roland DeGuio \\ INSA Graduate School of Science and Technology - LGeCO \\ 24 Boulevard de la Victoire, 67084Strasbourg Cedex FRANCE. \\ eltzer@yahoo.fr, roland.deguio@insa-strasbourg.fr
}

\begin{abstract}
Problem solving is a key activity for any innovative company. As the dialectical approach has shown its efficiency in problem solving, the starting point of our work is the use of contradiction in problem formulation stage. In this article, we analyse the possibility to use information brought by a Design of Experiments (DoE) to formulate a contradiction. Constraint Satisfaction Problem is employed to present information brought by the DoE. To use the dialectical approach, we define the concept of the Generalised Contradiction (GC). In the framework of DoE data, we show how to formulate a GC, and propose an algorithm able to identify OTSM-TRIZ System of Contradictions. Results and perspectives are discussed.
\end{abstract}

\section{Introduction}

Any manufacturing company often faces the need to solve problems: quality problems, industrialisation problems, design activity, etc. In this article we adress the domain of innovation problems. Solving an innovation problem means obtaining a product description detailed enough to manufacture it, [1]. Of course, this product should have precise properties (some of which are known at the beginning of the solving process). A part of innovation problems can be solved using an optimisation approach: the parameters of the product remain the same and one searches their best possible values, [2]. However, some problems cannot be solved by such an approach: the set of required properties returns an empty solution space. This means that the product has to be completely rethought, because no satisfying solution will be found by simply adjusting the parameters values. The product architecture has to be redefined. For such a solutionless problem, optimisation techniques are useless: another solving approach has to be proposed to the designer. Any problem solving process contains at least two phases: problem formulation and problem resolution,

Please use the following format when citing this chapter:

Eltzer, T., DeGuio, R., 2007, in IFIP International Federation for Information Processing, Volume 250, Trends in Computer Aided Innovation, ed. León-Rovira, N., (Boston: Springer), pp. 145-155. 
[3]. In this article, we focus on problem formulation and develop a new framework to formulate the problem of solutionless situations.

The dialectical approach has been developed by famous philosophers: Plato [4], Hegel, Marx, Mao Tse-Tung, [5]. The dialectical way to adress a problem has the three following bases: a thesis, its opposed antithesis, and the solution in which these two opposites can coexist. Thus, the core of the dialectical approach is the identification of a contradiction between a thesis and an antithesis. The dialectical way of formulating a problem is the formulation of such a contradition. Dialecticbased problem solving approaches have shown a significant efficiency in, among others, the following domains: information system design [6], manufacturing process design [7] or inventive technical problems [8]. In the third domain, a complete theory has been developed: TRIZ. Its development has been initiated by G. Altshuller. One of the main ideas is to use contradictions as a way to formulate problems. More than 50 years after the first publication on this problem solving approach, some evolutions have been proposed, among others: General Theory of Advanced Thinking (russian acronym OTSM), Theory of Development of a Strong Creative Personality (TRTL), Theory of the Evolution of Technological Systems (TRTS). It is in the border of OTSM-TRIZ that the idea of contradiction is the most clearly defined, [9]. The proposed System of Contradictions is inspired by the dialectical approach and artificial intelligence models. A schema representing the System of Contradictions (SC) is given Fig. 1. Facts concerning a classical laptop are used to illustrate the SC.

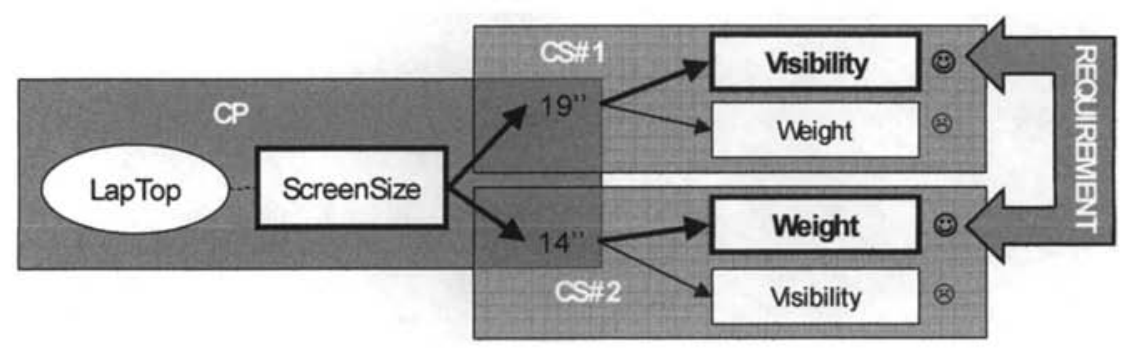

Fig. 1. System of Contradictions, applied to a laptop

The first component of a SC is the "requirement": two measured parameters, of which the value should belong to a predefined domain. The requirement describes the objective of the problem solving. The laptop weight and information visibility should be both satisfying. Weight should be low and visibility should be high. The second component of a SC is the "contradiction of a parameter": a parameter X should be assigned two different values. The screensize should be both 19" and 14". The remaining components of a SC are two "contradictions of a system": if one of the two possible values is assigned to $\mathrm{X}$, one measured parameter is satisfying, and the second one is not. CS\#1: if the laptop screensize is 19", then visibility is good but weight is bad. CS\#2: if the size is 14", then weight is good, but visibility is bad. Problem solving principles have been built for the SC: 13 principles to solve a contradiction of a system, 6 principles to solve a contradiction of a parameter [10]. Thus, we will investigate the possibility to use the SC model in a complex set of information. 
A SC is formulated between two pieces of information. For innovative design problems, the first available information source is the expert designer: a contradiction will be proposed on the basis of what the expert designer says. However, this information source has, among others, the following disadvantages: (1) it is difficult to extract tacit knowledge (2) an expert does not want to share everything (3) experts can be wrong (4) time required to gather enough and reliable information can be very long. Nowadays, other sources of informations are available especially because companies develop their own knowledge management activites, [11]. Part of our research activity investigates the possibility to use alternative information sources in the problem formulation phase, to complement or rectify expert knowledge. In this article, we focus on how to extract contradictions and SC out of a Design of Experiments.

\section{Design of Experiments}

The tools and techniques existing in the border of DoE can be grouped in two families which are modelling and optimisation. The first family of tools assists the pragmatic construction of mathematical models describing a measurable phenomenon, [12]. Parameters concerned by a DoE are either measurable or controlled. The controlled parameters are usually noted $X$ (the value is chosen) and the measured parameter is usually noted $Y$ (its value is only observed). Usually, the measured parameter describes a portion of the product requirement. A mathematical model is built on the basis of the set of achieved experiments. Experimental measures are usually listed in a chart (Table 1). One of the objectives of DoE tools is to obtain a realistic model with the minimum number of experiments. Robustness of the obtained model can be valuated using statistical calculations. The number of requested experiments can be reduced using Tagushi's method [13], fractionnary plan technique, or by removing some parameters based on the experts opinion. The second family of DoE techniques concerns the exploitation of the obtained mathematical model, [14]. The major kind of exploitation is the determination of requested controlled parameters values: given a required value of the measured variable, the mathematical model is used to find requested controlled parameters values. Different optimisation algorithms exist, but as we said in the introduction, we do address in this paper problems that cannot be solved by optimisation as no solution do exist within the given model. In this article, we consider that the domain of each controlled parameter is continuous. The value chosen during an experiment is one out of a continuous domain.

Let's consider as an example that will be used along the paper, a problem with two controlled parameters $X_{1}$ and $X_{2}$, influencing three variables $Y_{1}, Y_{2}$ and $Y_{3}$ simultaneously measured. The values of $X_{1}$ and $X_{2}$ must be chosen within the range $[-1,1]$. The requirement is that $Y_{1}, Y_{2}$ and $Y_{3}$ should be greater than 6 . We are looking for pairs $\left(\mathrm{X}_{1}, \mathrm{X}_{2}\right)$ that fit this requirement by performing a DoE, the result of which is given in Table 1. Four experiments have been done. 
Table 1. Example of DoE result

\begin{tabular}{llllll}
\hline $\operatorname{Exp~}^{\circ}$ & $\mathrm{X}_{1}$ & $\mathrm{X}_{2}$ & $\mathrm{Y}_{1}$ & $\mathrm{Y}_{2}$ & $\mathrm{Y}_{3}$ \\
\hline 1 & -1 & -1 & 10 & 10 & 0 \\
2 & -1 & +1 & 10 & 0 & 10 \\
3 & +1 & -1 & 0 & 10 & 10 \\
4 & +1 & +1 & 0 & 0 & 0 \\
\hline
\end{tabular}

The obtained mathematical model describing the effect of $X_{1}$ and $X_{2}$ on $Y_{1}, Y_{2}$ and $\mathrm{Y}_{3}$ is respectively:

$$
\begin{gathered}
Y_{1}=f_{1}\left(X_{1}, X_{2}\right)=-5 \cdot X_{1}+5 \\
Y_{2}=f_{2}\left(X_{1}, X_{2}\right)=-5 \cdot X_{2}+5 \\
Y_{3}=f_{3}\left(X_{1}, X_{2}\right)=-5 \cdot X_{1} \cdot X_{2}+5
\end{gathered}
$$

Fig. 2 provides a graphical representation of the three models of effects. $Y_{1}, Y_{2}$ and $Y_{3}$ are plotted versus both $X_{1}$ and $X_{2}$. In each of the three diagrams, the grey floor represents the possible values for $\left(X_{1}, X_{2}\right)$ and the grid represents the consequent value of the measured variable. The range of colors is linked to the value of the measured variable. The posed problem is solutionless with the table and the mathematical model. Indeed, whatever the value of $\left(\mathrm{X}_{1}, \mathrm{X}_{2}\right)$ within the allowed domain, there is at least one measured variable which does not satisfy the requirements. However, as each couple of measured variable can be simultaneously satisfied (for example, test $n^{\circ} 1$ shows this for $Y_{1}$ and $Y_{2}$ ), no SC among the parameters of the given models can be found in this example. In this paper, we do not investigate the possibility to change the problem definition to fit the SC pattern, as our purpose is to automatically extract contradictions from a given model.
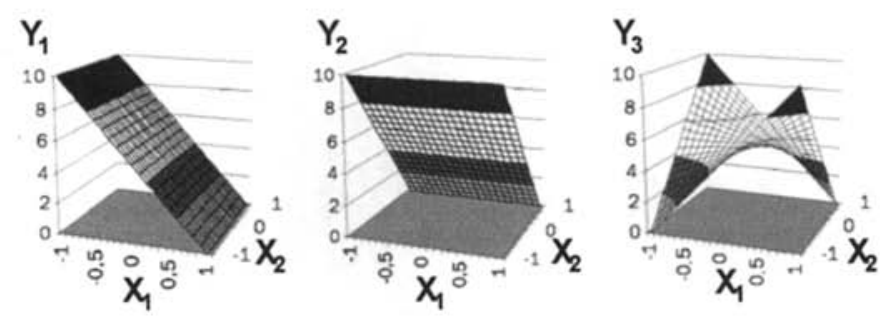

Fig. 2. Graphical representations of the relations among the five parameters

This simple case example shows that there is still a need for a problem formulation pattern, based on the dialectical approach, which could be used for any solutionless model. Table 2 lists our proposed criteria for this kind of problems. 
Table 2. Criteria to evaluate the proposed problem model

\begin{tabular}{|c|c|c|}
\hline${\text { Criteria }{ }^{\circ}}^{\circ}$ & Description & Comment \\
\hline $\mathrm{Cl}$ & $\begin{array}{l}\text { Existance of solving tools } \\
\text { that can be applied on the } \\
\text { problem decription }\end{array}$ & $\begin{array}{l}\text { This is an important criterion, as the goal of } \\
\text { problem formulation is not only description, but } \\
\text { mainly resolution. }\end{array}$ \\
\hline $\mathrm{C} 2$ & $\begin{array}{l}\text { The problem formulation } \\
\text { pattern describes a property } \\
\text { that can be found in any } \\
\text { problematic situation }\end{array}$ & $\begin{array}{l}\text { This second criterion directly emerged from the } \\
\text { case example which cannot be described using } \\
\text { OTSM-TRIZ model }\end{array}$ \\
\hline $\mathrm{C} 3$ & $\begin{array}{l}\text { The problem formulation } \\
\text { pattern describes a property } \\
\text { that is absent of any solvable } \\
\text { problem }\end{array}$ & $\begin{array}{l}\text { The dialectical approach efficiency has only been } \\
\text { shown in solutionless problems. }\end{array}$ \\
\hline $\mathrm{C} 4$ & $\begin{array}{l}\text { The problem is completely } \\
\text { described with a single } \\
\text { contradiction }\end{array}$ & $\begin{array}{l}\text { If this is not true, the problem solver will have to } \\
\text { select one of them, and, even if the selected } \\
\text { contradiction is solved, the problem can remain } \\
\text { unsolved. }\end{array}$ \\
\hline $\mathrm{C} 5$ & $\begin{array}{l}\text { The problem formulation } \\
\text { pattern provides a synthetic } \\
\text { description of the problem }\end{array}$ & $\begin{array}{l}\text { In the other case, it might be difficult for humans } \\
\text { to handle the description }\end{array}$ \\
\hline
\end{tabular}

\section{Constraint Satisfaction Problems}

\subsection{Definition}

Montanari introduced the constraint satisfaction problem (CSP) model in [15]. Any CSP comprises:

- a set of $n$ variables $V^{\prime}=\left(V_{1}, \ldots, V_{n}\right)$. A value of $V_{k}$ is noted $v_{k}$. Therefore, $v^{\prime}$ is defined as the tuple $\left(\mathrm{v}_{1}, . ., \mathrm{v}_{\mathrm{n}}\right)$;

- a set of domains $D^{\prime}=\left(D_{1}, \ldots, D_{n}\right)$ where $D_{i}$ is the finite set of possible values for $V_{i}$;

- a set of constraints $C^{\prime}=\left(C_{1}, . ., C_{j}\right)$ between the existing variables. $A$ constraint $C$ on a subset of $V$ ' specifies the allowed combination of values for the variables.

A solution to a CSP is an assignment of a value from its domain to every variable, in such a way that all constraints are satisfied at once. A constraint is not satisfied when the assignment of the variables is not allowed by the constraint. A CSP is solvable if at least one solution exists. Two analyses can be done on a CSP: find one solution, or find all solutions. These two objectives gave birth to different solving algorithms, [16].

Therefore, controlled and measured parameters of a DoE simply become "variables" in a CSP. Controlled parameters are grouped in $X '=\left(X_{1}, ., X_{m}\right)$ and measured parameters are groupped in $Y^{\prime}=\left(Y_{1}, . ., Y_{p}\right)$. Of course, $V^{\prime}=X^{\prime} U Y^{\prime}$. Domains of controlled parameters are grouped in $D_{X}^{\prime}=\left(D_{X 1}, \ldots D_{X m}\right)$ and domains of measured parameters are grouped in $D_{Y}^{\prime}$. An assignment of a value to each control parameter is named $x^{\prime}=\left(x_{1}, . ., x_{m}\right) \in D^{\prime}{ }_{X}$. Concerning the measured variable $Y_{k}$, the domain $D_{Y k}$ is the set of satisfying values. In the case example, as $Y_{1}, Y_{2}$ and $Y_{3}$ should be not less than $6, D_{Y 1}=D_{Y 2}=D_{Y 3}=[6 ;+\infty[$. The solution is not satisfying if one of them is out of 
this domain. For each parameter $Y_{k}$, its assigned value $y_{k}$ should belong to $D_{Y k}$. Concerning the controlled variables, the domain is the complete range of possible values. In the case example, the domain for $\mathrm{X}_{1}$ and $\mathrm{X}_{2}$ is the same. $\mathrm{D}_{\mathrm{X}_{1}}=\mathrm{D}_{\mathrm{X} 2}=[-1 ;+1]$. At this stage, the CSP model representing the case example is defined by:

- the set of five variables. $V^{\prime}=X^{\prime} U Y^{\prime}=\left(X_{1}, X_{2}, Y_{1}, Y_{2}, Y_{3}\right) . \quad X^{\prime}=\left(X_{1}, X_{2}\right)$; $\mathrm{Y}^{\prime}=\left(\mathrm{Y}_{1}, \mathrm{Y}_{2}, \mathrm{Y}_{3}\right)$;

- the set of domains: $D^{\prime}=\left(D_{Y 1}, D_{Y 2}, D_{Y 3}, D_{Y 4} D_{X 1}, D_{X 2}\right)$.

\subsection{Design of Experience and Constraints}

In this section we show in which shape the result of a DoE can be found in a CSP. The DoE often ends by the definition of a mathematical model, linking the controlled parameters and one measured parameter. Thus, this mathematical model directly specifies the allowed combination of values for these parameters. In fact, the equation describing this mathematical model defines the constraint linking the controlled parameters and the measured variable. Therefore, as we have made the hypothesis that the values of the controlled parameters are one out of a continuous range, the set of achieved experiments allows the definition of a constraint in intension. This constraint links p variables, and only one of them is measured.

In our case example, as there are three measured variables, three constraints are defined: $C^{\prime}=\left\{C_{1}, C_{2}, C_{3}\right\} . C_{1}, C_{2}$ and $C_{3}$ contraints are defined by Eq.1, Eq2 and Eq.3. The CSP of the case example is now completely defined by $V^{\prime}, D^{\prime}$ and C'.

\section{The proposed framework: the Generalised Contradiction}

\subsection{Definition}

Applying the dialectical approach on a solutionless problem comes to present it as an opposition. The very first detected opposition is between the required values of the set of measured parameter and the possible values of the set of controlled parameters. "The problem is unsolvable" means that there is no way to simultaneously create a satisfying value for each measured parameter. Each time the controlled parameters are tuned to satisfy $Y_{i}$, at least one other measured parameter $Y_{j,(i \neq j)}$ is not satisying. This means that the set of controlled parameter values able to satisfy $Y_{i}$ and the set of values able to satisfy $Y_{j}$ have no intersection. Let us define $S_{Y i}$ as the set of values of $X^{\prime}=\left(X_{1}, . ., X_{m}\right) \in D_{X}$ ' which are able to guarantee that $Y_{i}$ is satisfying (Eq.1). It should be noted that if there is one, two or three controlled parameters, $\mathrm{S}_{\mathrm{Yi}}$ geometric interpretation is respectively a line, a surface, or a volume. We define the Generalised Contradiction as the property of $Y$ ' detailed in Eq.5: there

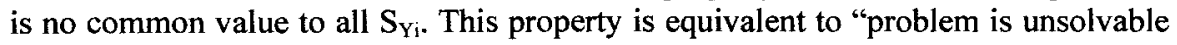
with the given model", as proved by the two following demonstations:

- if the property described by Eq.5 is wrong, then it means that it exists an assignment $x^{\prime}$ to $X^{\prime}$ which belongs to any $S_{Y_{i}}$. This means that $x^{\prime}$ creates a satisfying value of any $Y_{i}$. This means that the problem is not solutionless: if $\mathrm{X}^{\prime}=\mathrm{X}^{\prime}$, each $\mathrm{Y}_{\mathrm{i}}$ is satisfying. Hence, if Eq.5 is true, the problem is solutionless; 
- if the problem is not solutionless, then it exists an assignment $x^{\prime}$ to $X^{\prime}$ able to create a satisfying value to each $Y_{i}$. Hence, by definition, $x^{\prime}$ belongs to any $S_{Y_{i}}$. Thus, the intersection is not empty. Hence, if the problem is solutionless, Eq.5 is true.

$$
\begin{gathered}
S_{Y_{i}}=\left\{x^{\prime} /\left(y_{i}=f_{i}\left(x^{\prime}\right) \in D_{Y_{i}}\right) A N D\left(x^{\prime} \in D^{\prime}{ }_{X}\right)\right\} \\
\bigcap_{i / Y_{i} \in Y^{\prime}} S_{Y_{i}}=\varnothing
\end{gathered}
$$

Let us illustrate this property on the case example. As there are three measured parameters, there are three sets $\mathrm{S}_{\mathrm{Y} 1}, \mathrm{~S}_{\mathrm{Y} 2}$ and $\mathrm{S}_{\mathrm{Y} 3}$. As there are two controlled parameters, each of these domains has two dimensions, i.e it is a surface. Fig. 3(a) shows each of these three surfaces in the square of the possible values $x^{\prime}$ of $X^{\prime}=\left(X_{1}, X_{2}\right)$. One can notice the three following facts: (1) $S_{Y_{1}} \cap S_{Y_{2}}$ is not an empty set (the bottom left square), it means that it is possible to reach a satisfying value for both $\mathrm{Y}_{2}$ and $\mathrm{Y}_{3}$ simultaneously; (2) neither $\mathrm{S}_{\mathrm{Y}_{1}} \cap \mathrm{S}_{\mathrm{Y} 3}$ nor $\mathrm{S}_{\mathrm{Y} 2} \cap \mathrm{S}_{\mathrm{Y} 3}$ are empty sets, which finally means that each couple of measured variable can be reached simultaneously; (3) $\mathrm{S}_{\mathrm{Y}_{1}} \cap \mathrm{S}_{\mathrm{Y}_{2}} \cap \mathrm{S}_{\mathrm{Y}}=\emptyset$, which means that the problem is solutionless.

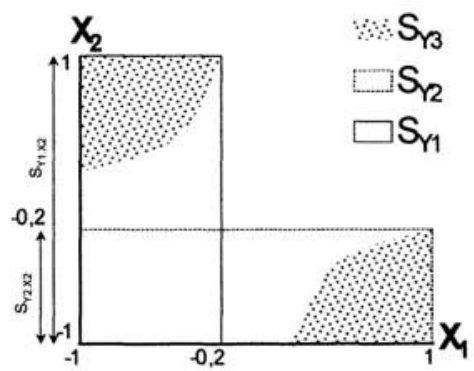

(a)

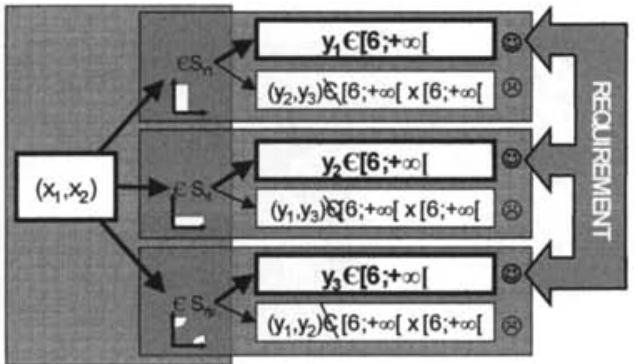

(b)

Fig. 3. (a) SY1 and SY3 (b) Possible GC graphical representation

In comparison, we can say that the System of Contradictions is concerned only by the intersection of a couple of domains $\mathrm{S}_{\mathrm{Yi}_{\mathrm{i}}}$ and $\mathrm{S}_{\mathrm{Y}_{\mathrm{j}}}$ in one of their dimensions. Therefore, if we define $S_{Y_{i} X k}$ as the set of values $x_{k}$ which can satisfy $Y_{i}(E q 6)$, we can say that the $\mathrm{SC}$ is based on the fact that $\mathrm{S}_{\mathrm{Yi} X \mathrm{Xk}}$ and $\mathrm{S}_{\mathrm{Yj}, \mathrm{Xk}}$ have no common value. However, we have shown that this condition is not equivalent to the absence of solution. In our case example, we have: $\mathrm{S}_{\mathrm{Y}_{1} \mathrm{X}_{2}}=[-1 ; 1]$ and $\mathrm{S}_{\mathrm{Y} 2} \times \mathrm{X}_{2}=[-1 ;-0,2]$. The intersection is not empty because if $x_{2} \in[-1 ;-0,2]$ we can satisfy both $Y_{1}$ and $Y_{2}$. However, the impossibity to simultaneously satisfy $\mathrm{Y}_{3}$ is not seen.

$$
S_{Y_{i}, X_{k}}=\left\{x_{k} / \exists\left(x_{1}, . ., x_{k-1}, x_{k+1}, . ., x_{m}\right) /\left(\left(x_{1}, . ., x_{m}\right) \in S_{Y_{i}}\right)\right\}
$$

In Fig. 3(b), we use the SC scheme to represent the GC of the case example:

- the requirement is that both $Y_{1}, Y_{2}$ and $Y_{3}$ are satisfying; 
- $\left(\mathrm{x}_{1}, \mathrm{x}_{2}\right)$ shoud belong to $\mathrm{S}_{\mathrm{Y} 1}, \mathrm{~S}_{\mathrm{Y} 2}$ and $\mathrm{S}_{\mathrm{Y} 3}$;

- when $\left(x_{1}, x_{2}\right)$ belongs to $S_{Y 1}, Y_{1}$ is satisfying but at least one variable of $\left(Y_{2}, Y_{3}\right)$ is unsatisfying; when $\left(x_{1}, x_{2}\right)$ belongs to $S_{Y 3}, Y_{3}$ is satisfying but not $\left(Y_{1}, Y_{2}\right)$; when $\left(x_{1}, x_{2}\right)$ belongs to $S_{Y_{2}} Y_{2}$ is satisfying but not $\left(Y_{1}, Y_{3}\right)$.

\subsection{Algorithm for getting SC}

The following algorithm searches possible SC. The first step consists in calculating each $\mathrm{S}_{\mathrm{Yi}}$, then the i2-loop analyses each possible couple $\left(\mathrm{S}_{\mathrm{Yi}}, \mathrm{S}_{\mathrm{Y}_{\mathrm{j}}}\right)$ and checks the intersection in each dimension (i3-loop). The output of this algorithm is the exhaustive list of SC. As two calculations have to be done for any triplet of $\left(X_{k}, Y_{i}, Y_{j}\right)$, the total number of calculation is $\operatorname{Card}\left(Y^{\prime}\right)+2 \cdot \operatorname{Card}\left(X^{\prime}\right) \cdot 2^{\operatorname{Card}\left(Y^{\prime}\right)}$.

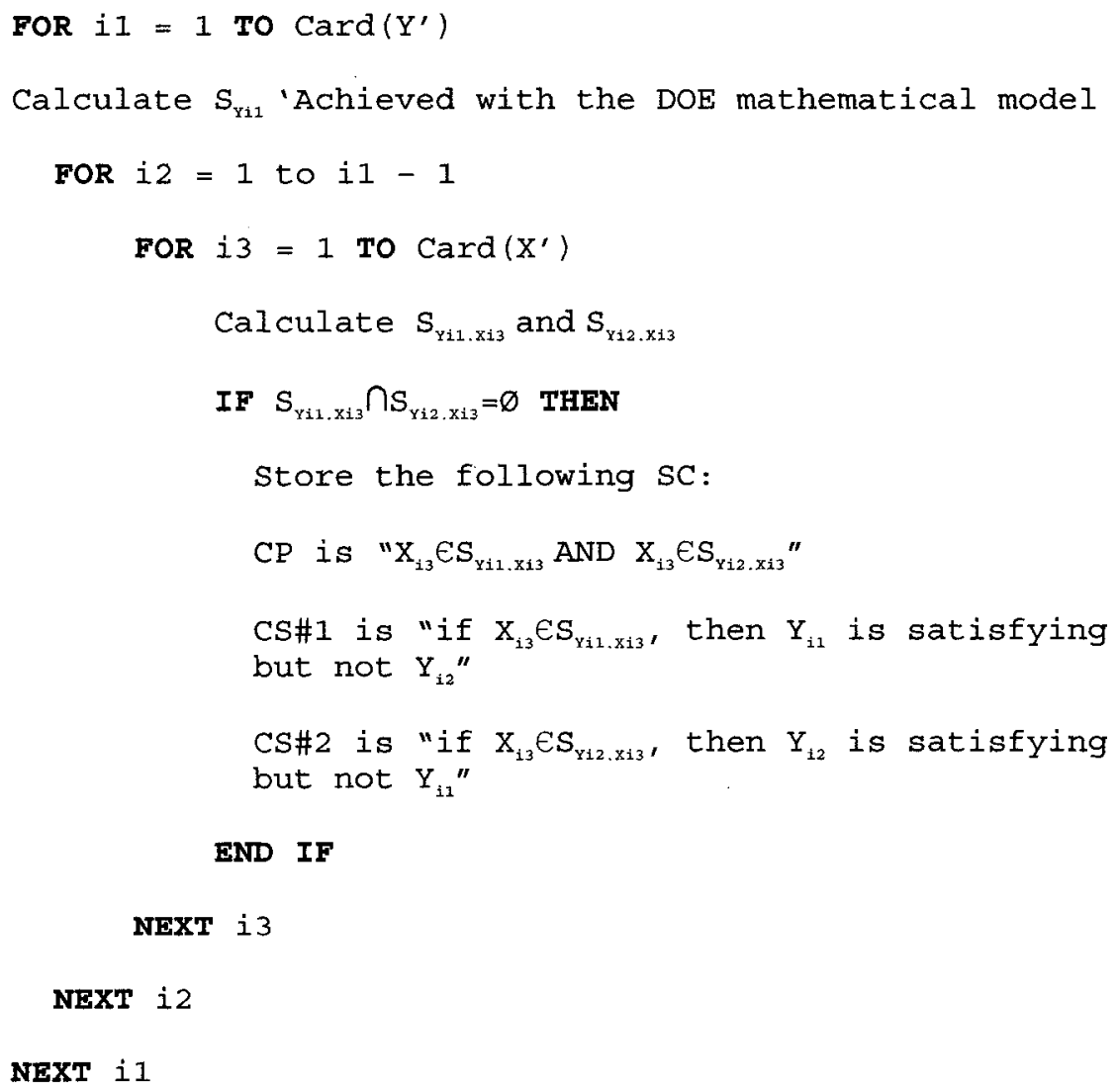

\section{Discussion}

First, we briefly compare the strong and weak points of our contribution and of the System of Contradictions of OTSM-TRIZ according to the five criteria of Table 2. 
C1: different solving principles have been adapted from TRIZ to OTSM-TRIZ. We have not yet developed the corresponding solving mechanisms for the GC framework. C2: the simple case example we developed is a solutionless problem. However, the SC could not describe it. We demonstrated that the GC basic property (Eq5) fits this criterion. C3: this is true for both the SC and the GC. C4: the System of Contradictions is based on the property of a triplet $\left(X_{k}, Y_{i}, Y_{j}\right): S_{Y_{i . X k}} \cap S_{Y_{j} . X k}=\varnothing$. In problems concerned by many measured and controlled parameters, this property can be found for more than one triplet, [17], [18]. This raises the following questions: which one should be chosen? how to navigate in and manage many contradictions? The GC is based on a property of $\mathrm{Y}^{\prime}$ (defined in Eq5). This property is unique, as, for a given problem, there is a single $Y^{\prime}$. C5: the System of Contradictions is very synthetic, as there are only three parameters. However, one can miss a lot of useful information. Describing a $\mathrm{GC}$ requires to list each $\mathrm{S}_{\mathrm{Yi}}$, which can be less synthetic. We can consider the GC is a generalization of the SC along these five criteria.

In this paper, we have addressed the problem of solutionless problems and proposed the GC framework to support the formulation phase. In the borders of constraints based problem modelling, solutionless problems are named "overconstrained problems": the constraints are so numerous that no satisfying value assignment is allowed, [19]. To face such a solutionless situation, the main current principle is known as "constraint relaxation". Constraints are simply removed one by one to open the solution space. A lot of research work concerns constraints relaxation order. Two main drawbacks of constraint relaxation are (1) if the constraint represents a scientific law (this is the case for the DoE mathematical model), constraint relaxation has no sense and (2) the objectif may not be reached, ie the product won't have each required feature. Our research results bring the first brick to build a new solving strategy. This strategy will consist in, first, formulating a $\mathrm{GC}$ to describe the problem and, second, applying solving mechanisms to this model. Extending the TRIZ solving mechanisms at the level of our GC framework is part of our current research. The second contribution in this domain is that the GC can link optimisation design tasks and inventive design tasks. Up to now a design problem had to be treated either with optimisation techniques (Operations Research algorithms to search for a solution in a CSP, [2]), or with inventive approaches (like TRIZ, for simple inventive tasks, [8]). No single information support could be used for these two tasks. Thanks to the proposed GC framework, information can be formalised in a CSP model and treated with both optimisation algorithms and inventive procedures. Furthermore, as CSP modelling is very generic and can be applied to numerous domains, the GC pattern can be applied to analyse other sources of corporate data, [11].

\section{Conclusion}

In this article, we have addressed the description of solutionless problems using the dialectical philosophy. We have considered that Design of Experiments can be used as an information source. The General Contradiction has been proposed to bypass the fact that, in complex cases, the System of Contradictions of OTSM is not equivalent to the absence of a solution. The Generalised Contradiction describes the fact that 
satisfying one requirement degradates at least another one. The Generalised Contradiction considers intersections of sets which dimensions equals the number of controlled parameters. One of the key advantages of the $\mathrm{GC}$ is the possibility to represent any complicated problems with a dialectical approach.

The first research direction consists in proposing problem solving mechanisms, based on those developed developed in TRIZ and OTSM-TRIZ, to fit the GC framework. The second research direction concerns the description of the GC. The fact that the intersection of SYi is empty can be described in many different ways: one domain is empty, one (or more) couple of domains has no intersection, one (or more) triplet of domains has no intersection, etc. One research activity aims now at finding which combination of parameters and domains should be used to provide a comprehensive and synthetic description of the GC. The third research perspective is the development of a more efficient algorithm to provide a comprehensive GC description. The first possibility is to use already existing CSP algorithms to provide automatic contradiction identification. The key question to answer is the equivalence between contradictions and consistencies, as defined in CSP modelling. The second possibility is searching only those contradictions separating the unreachable desired system from the today best reachable ones.

\section{References}

1. W. BEITZ and G. PAHL, Engineering design - A systematic approach (Springer, 1996).

2. F. Hillier and G. Lieberman, Introduction to Operations Research, (McGraw-Hill, 2005).

3. S. Easterbrook, E. Beck, J. Goodlet et al, A survey of empirical studies of conflict, in CSCW: Cooperation or conflict (Springer, 1993).

4. S. Scolnicov (traducer) Plato's Parmenides (University of California Press, LA, 2003)

5. M. Tsetung, On Contradiction, in Selected Works, 311-347 (Foreign Languages Press, Peking, 1967).

6. V. Goepp, F. Kiefer and F. Geiskopf, Design of information system architectures using a key-problem framework, Computers in Industry, 57(2), 189-200, (2006).

7. F. Geiskopf, Formalisation et exploitation des contraintes Produit/Process pour la conception de systèmes de production - application à l'Usinage Grande Vitesse, $\mathrm{PhD}$ thesis, (ULP, Strasbourg France, 2004).

8. G. Altshuller, Creativity as an exact science, (Gordon and Breach Publishers, 1988).

9. N. Khomenko, R. De Guio and D. Cavallucci, Enhancing ECN's abilities to adress inventive strategies using OTSM-TRIZ, Int. J. Collaborative Engineering, (unpublished).

10. N. Khomenko, Introduction to OTSM-TRIZ. (Lectures of Advanced Master of Innovative Design, INSA Strasbourg, 2005).

11. N. Bontis, N. Dragonetti, K. Jacobsen and G. Roos, The Knowledge Toolbox: A review of the tools available to measure and manage intangible resources, European Management Journal, 17(4), 391-402 (1999).

12. D. C. Montgomery, Design and Analysis of Experiments, (Wiley-Interscience, 2004)

13. R. K. Roy, Design of Experiments Using The Taguchi Approach: 16 Steps to Product and Process Improvement, (Wiley-Interscience, 2001)

14. G. B. Dantzig, Origins of the simplex method, in A history of scientific computing, 141151 (Reading, MA, 1990).

15. Montanari, Network of constraints: fundamental properties and applications to picture processing, Information science, 7, 95-132 (1974).

16. V. Kumar, Algorithms for constraint-satisfaction problems: a survey, AI Magazine, 13(1), $32-44$ (1992) 
17. D. Cavallucci and N. Khomenko, From TRIZ to OTSM-TRIZ: Addressing complexity challenges in inventive design, Int. J. of Product Development (unpublished).

18. E. Freuder and R. Wallace, Partial Constraint Satisfaction, Artificial Intelligence 58, 21$70,(1992)$

19. T. Eltzer, D. Cavallucci, N. Khomenkho, P. Lutz, E. Caillaud, Problem Formulating for Inventive Design Application to Injection Molding Technology, in Advances in Design, Chapter 3.6, (Springer, 2006) 